\title{
Potenciais reações adversas relacionadas a antipsicóticos ou antidepressivos e fármacos associados em pacientes do Centro de Atenção Psicossocial (CAPS) "Esperança" de Recife
}

\author{
Mirela Galvão de Barros $^{\mathrm{a}}$, Filipe Silveira Duarte $\mathrm{b}^{\mathrm{b}^{*}}$ \\ àDepartamento de Ciências Farmacêuticas, Centro de Ciências da Saúde, \\ Universidade Federal de Pernambuco, Recife, PE, Brasil \\ ${ }^{b}$ Departamento de Fisiologia e Farmacologia, Centro de Biociências, \\ Universidade Federal de Pernambuco, Recife, PE, Brasil
}

\section{Histórico do Artigo \\ Recebido em: \\ 28/02/2019 \\ Aceito em: \\ 04/02/2020}

\section{Palavras-chave:}

Reações adversas; antidepressivos; antipsicóticos; politerapia; Serviços de Saúde Mental
Keywords:

Adverse reactions; antidepressants; antipsychotics; Polytherapy; Mental Health Services

\begin{abstract}
RESUMO
Objetivou-se identificar os principais antipsicóticos e antidepressivos utilizados em um Centro de Atenção Psicossocial (CAPS) e caracterizar o perfil de pacientes submetidos ao tratamento em politerapia analisando os riscos potenciais das reações adversas. Trata-se de uma pesquisa transversal, descritiva com abordagem qualitativa e quantitativa, realizada no CAPS "Esperança" de Recife (Pernambuco). Participaram deste estudo usuários admitidos entre janeiro de 2013 e junho de 2016. Uma triagem inicial de 155 prontuários médicos foi realizada visando identificar o perfil do usuário quanto ao diagnóstico clínico e medicamentos utilizados. Na segunda etapa, 51 pacientes em regime de tratamento em politerapia foram entrevistados visando identificar as principais associações medicamentosas e as reações adversas mais relatadas. A média da idade dos pacientes foi de 44 anos (91 femininos e 64 masculinos) com transtornos mentais de alta gravidade, predominando a esquizofrenia $(45,8 \%)$ e transtornos de humor $(31,6 \%)$. As classes medicamentosas mais utilizadas foram os antipsicóticos (40\%), com destaque para levomepromazina e risperidona, seguido dos antidepressivos (14\%) como fluoxetina e citalopram. A análise identificou 13 tipos de associações medicamentosas, entre as quais se destacaram aquelas constituídas por 1 antipsicótico típico + 1 antipsicótico atípico com prometazina e/ou biperideno (17,6\%), 2 ou mais antipsicóticos típicos com prometazina e/ou biperideno $(11,8 \%)$ e 1 antipsicótico (típico ou atípico) com prometazina e/ou biperideno associado a 1 antidepressivo (11,8\%). As reações adversas mais relatadas foram os sintomas anticolinérgicos $(64,7 \%)$, ganho de peso $(56,9 \%)$ e sintomas extrapiramidais $(50,9 \%)$. Os resultados apontam para o potencial elevado de reações adversas no âmbito do CAPS "Esperança", possivelmente relacionadas ao regime de tratamento em politerapia.
\end{abstract}

Potential adverse reactions related to antipsychotics or antidepressants and associated drugs in patients at the "Esperança" Psychosocial Attention Center (CAPS) in Recife

\section{ABSTRACT}

The aim of this study was to identify the main antipsychotics and antidepressants drugs used in a Psychosocial Attention Care Center (CAPS), and to characterize the profile of patients under polytherapy treatment regimens analyzing the potential risks of adverse reactions. This is a crosssectional, descriptive study with a qualitative and quantitative approach, carried out at the "Esperança" CAPS in Recife (Pernambuco). The study included healthcare users admitted between January 2013 and June 2016. An initial screening of 155 medical records was carried out aiming to identify the profile of users as to their clinical diagnosis and main medications used. In the second stage, 51 patients under polytherapy regimens were interviewed in order to identify the main types of drugs combinations used and the adverse reactions most frequently reported. The mean age of the users was 44 years old (91 female and 64 male) with high severity of mental disorders, with schizophrenia predominating $(45.8 \%)$, followed by mood disorders $(31.6 \%)$. The most used medication class was antipsychotics (40\%), with emphasis on levomepromazine and risperidone, followed by antidepressants (14\%) such as fluoxetine and citalopram. The analysis identified 13 different types of drugs combinations, with the most frequent being constituted by 1 typical antipsychotic +1 atypical antipsychotic with promethazine and/or biperiden (17.6\%), 2 or more antipsychotics with promethazine and/or biperiden $(11.8 \%$ ), and 1 antipsychotic (typical or atypical) with promethazine and/or biperiden associated with 1 antidepressant (11.8\%). The most commonly reported adverse reactions were anticholinergic symptoms (64.7\%), weight gain (56.9\%) and extrapyramidal symptoms $(50.9 \%)$. The results point to the high potential of adverse reactions in the "Esperança" CAPS, possibly related to the polytherapy treatment regimen.

\footnotetext{
* Autor correspondente: filipeduarteufpe@gmail.com, filipe.sduarte@ufpe.br (Duarte F.S.)
} 


\section{Introdução}

Os transtornos psiquiátricos vêm aumentando em taxas expressivas na população mundial, em parte devido às mudanças ocorridas no contexto socioeconômico-cultural. No Brasil, as taxas de prevalência variam entre 20 a $56 \%$ para os diferentes tipos de transtornos mentais (1). A esquizofrenia e os transtornos depressivos merecem destaque no cenário da psiquiatria pela elevada incapacitação na esfera socio-ocupacional e aumento do risco de suicídio, ocupando uma parcela significativa da carga global de saúde (2).

Dados epidemiológicos da Organização Mundial de Saúde (OMS) apontam a existência de 322 milhões de pessoas acometidas por algum tipo de transtorno depressivo no mundo, com o número de casos de depressão aumentando em 18\% entre 2005 e 2015 (2). No Brasil, a depressão atinge $5,8 \%$ da população brasileira $(1,2)$. Em contrapartida, a esquizofrenia afeta 23 milhões de pessoas no mundo, com uma prevalência de 1\% no Brasil e América Latina $(1,2)$ e, no geral, tem um curso evolutivo progressivo e de pior prognóstico em relação à depressão (3). Ambas as doenças mentais estão associadas ao grande sofrimento psíquico e comprometem consideravelmente o funcionamento global do paciente, sendo responsabilizadas pela maioria das internações psiquiátricas (3). Considerando este grave problema de saúde pública, o acesso aos serviços de saúde e psicossocial tornam-se imperativos, uma vez que os mesmos são capazes de fornecer aos seus usuários o devido tratamento e suporte social necessário.

Os Centros de Atenção Psicossocial (CAPS) são serviços de saúde constituídos por uma equipe multidisciplinar que atuam prioritariamente em atendimento a pacientes com transtornos mentais, visando a sua reabilitação e reintegração social (4). Dentre os tratamentos disponíveis na clínica e utilizados nos CAPS, encontra-se a terapia farmacológica, que consiste na utilização de medicamentos que permitem controlar a sintomatologia subjacente aos transtornos psiquiátricos (5). Entretanto, o uso inadequado dos medicamentos prescritos, sobretudo em regimes de tratamento em politerapia, assim como a longa duração do tratamento e o desconhecimento sobre suas reações adversas podem influenciar negativamente a adesão do usuário ao tratamento (6).

Os antidepressivos e antipsicóticos se destacam entre os medicamentos mais prescritos nas unidades dos CAPS. Apesar da comprovada eficácia, estes fármacos são responsáveis por induzir uma variedade de reações adversas que, por vezes, levam o paciente a abandonar o tratamento ou fazê-lo da maneira incorreta (6). Ao negligenciar-se o tratamento com estes medicamentos sem a devida identificação e notificação das reações adversas, o usuário se torna vulnerável à recidiva, aumentando o período de internação psiquiátrica e elevando os custos assistenciais $(5,6)$.

Para o tratamento dos transtornos depressivos no âmbito dos CAPS, existem diversas opções de fármacos, entre elas a categoria dos inibidores da monoaminoxidase (IMAOs), os antidepressivos tricíclicos (ATCs) e os inibidores seletivos de recaptação de serotonina (ISRS) (5-7). Por outro lado, o tratamento da esquizofrenia e transtornos psicóticos relacionados se dá primariamente através do uso de medicamentos antipsicóticos (5,8-9). Os primeiros descobertos e utilizados na prática clínica foram os antipsicóticos de $1^{\text {a }}$ geração (clássicos, tradicionais ou típicos), porém por provocarem graves reações adversas de natureza motora oriundas do antagonismo predominante de receptores dopaminérgicos- $\mathrm{D}_{2}$, começaram a ser substituídos pelos antipsicóticos de $2^{\mathrm{a}}$ geração (atípicos), que agem preferencialmente antagonizando os receptores serotoninérgicos 5$\mathrm{HT}_{2 \mathrm{~A}} \mathrm{e}$, em menor proporção, os receptores dopaminérgicos- $\mathrm{D}_{2}$, por isso, estes últimos apresentam menor potencial para induzir reações adversas neurológicas em relação aos primeiros. Enfatiza-se que tais drogas têm nas reações adversas os principais responsáveis 
pela descontinuação e baixa adesão terapêutica, redução da qualidade de vida, da interação e adaptação social dos pacientes (9).

Através do acompanhamento farmacoterapêutico de pacientes institucionalizados no âmbito dos CAPS e conhecendo-se as potenciais reações adversas ocasionadas por medicamentos de amplo uso na prática clínica, o profissional farmacêutico terá sua contribuição direta no que tange à orientação de seus pacientes quanto ao uso correto dos medicamentos, permitindo, assim, trazer maior qualidade de vida aos usuários de serviços de saúde mental (10-13). Diante do exposto, o presente estudo propôs identificar os principais antipsicóticos e antidepressivos mais prescritos no âmbito do CAPS "Esperança de Recife", caracterizar o perfil de pacientes submetidos ao regime de tratamento com antipsicóticos ou antidepressivos e fármacos associados, bem como analisar as potenciais reações adversas nesta população específica.

\section{Material e métodos}

Trata-se de uma pesquisa de ordem transversal, descritiva com abordagem qualitativa e quantitativa, realizada no CAPS "Esperança" de Recife (PE) entre os meses de março a junho de 2016. Neste período, uma coleta de dados e análise de prontuários de pacientes psiquiátricos foi realizada visando identificar as principais condutas terapêuticas envolvendo antidepressivos ou antipsicóticos, a fim de estabelecer o perfil das reações adversas ocasionadas por estas categorias de fármacos e discutir suas consequências. Participaram deste estudo pacientes psiquiátricos admitidos na instituição entre janeiro de 2013 e junho de 2016. Para a coleta de dados, foi realizada uma análise de 155 prontuários médicos contendo a identificação dos pacientes, as variáveis sociodemográficas, o diagnóstico clínico de acordo com a Classificação Internacional de Doença (CID-10), bem como os medicamentos utilizados. Após esta triagem e traçar o perfil farmacoterapêutico dos usuários do CAPS, foram selecionados 51 pacientes psiquiátricos para uma entrevista a partir de critérios de inclusão e exclusão. Pacientes fazendo uso de associações medicamentosas contendo pelo menos um antidepressivo ou um antipsicótico foram incluídos nesta etapa (informações obtidas pela fonte documental e confirmadas durante a entrevista). Estabeleceu-se como critérios de exclusão os pacientes que não se enquadraram no critério de inclusão e aqueles sem condições cognitivas de participarem da entrevista. Um questionário estruturado com questões abertas e fechadas, previamente elaborado pelos autores, foi aplicado a cada paciente, e contemplando variáveis relacionadas ao tratamento (aderência, presença de reações adversas) e à vida pessoal (trabalho, atividade física, uso de álcool, tabagismo, participação em oficinas terapêuticas fornecidas pelo CAPS, autopercepção do estado de saúde e a satisfação do paciente quanto às orientações fornecidas pelo CAPS sobre sua condição clínica e tratamento). Para o cálculo da amostragem, foi utilizada a fórmula $\mathrm{n}=\mathrm{N} /\left(\mathrm{E}_{\mathrm{o}}^{2} \times \mathrm{N}\right)+1$, onde $n$ representou o tamanho da amostra, $N$ o número de elementos da população e $E_{0}$ o erro amostral. Para este estudo foi considerado o $\mathrm{N}=90$ e $\mathrm{E}_{0}=10 \%$, correspondendo à amostragem de $\mathrm{n}=47$. Como foram realizadas 51 entrevistas, a amostragem utilizada nesta etapa foi considerada aceitável em relação à mínima exigida para este tipo de estudo ( $\mathrm{n} \geq 47$ ). Os dados foram analisados por meio de estatística descritiva. Os requisitos quanto à confidencialidade e ao sigilo das informações foram cumpridos de acordo com as determinações da resolução nº 466/2012, do Comitê de Ética do Conselho Nacional de Saúde (14). A presente pesquisa foi aprovada pelo Comitê de Ética em Pesquisa (CEP) da Universidade Federal de Pernambuco (UFPE), sob o número de registro 49954315.7.0000.5208. 


\section{Resultados}

Dos 155 prontuários médicos de pacientes submetidos a tratamento psiquiátrico no CAPS "Esperança", a análise identificou 91 pacientes do sexo feminino e 64 do sexo masculino, correspondendo a $59 \%$ e $41 \%$, respectivamente. A faixa etária geral variou de 18 a 70 anos, com uma média de 44 anos. A Figura 1 mostra a relação dos diagnósticos clínicos de acordo com a CID-10 e das principais classes de medicamentos utilizadas pelos pacientes. A análise dos prontuários mostrou um predomínio da esquizofrenia e transtornos psicóticos relacionados (F20-29), correspondendo a 45,8\%, seguido dos transtornos de humor (F30-39) com uma prevalência de 31,6\% (Figura 1A). A classe medicamentosa mais prescrita foi a dos antipsicóticos (40\%), seguido dos antidepressivos e ansiolíticos benzodiazepínicos, ambos dos quais correspondendo a 14\% (Figura 1B).
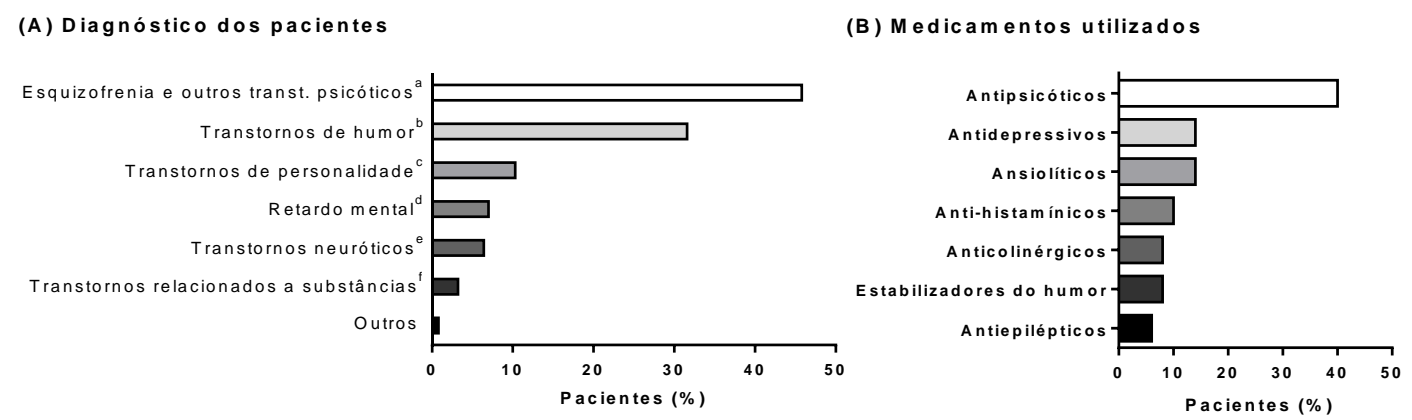

Figura 1 - Relação dos diagnósticos clínicos (A) e das classes de medicamentos utilizadas (B), obtida a partir dos prontuários médicos de 155 pacientes psiquiátricos admitidos no CAPS "Esperança” de Recife (PE), no período entre janeiro de 2013 e junho de 2016.

a,b,c,d,e,f Classificação dos diagnósticos psiquiátricos de acordo com a CID-10. ${ }^{a}$ Esquizofrenia, transtornos esquizotípicos e transtornos delirantes (F20-F29), ${ }^{\text {b }}$ Transtornos do humor [afetivos] (F30-F39), ${ }^{c}$ Transtornos de personalidade e do comportamento do adulto (F60-F69), ${ }^{\mathrm{d}}$ Retardo mental (F70-F79), ${ }^{\mathrm{e}}$ Transtornos neuróticos, transtornos relacionados com o "stress" e transtornos somatoformes (F40-F48), ${ }^{\mathrm{f}}$ Transtornos mentais e comportamentais devidos ao uso de substância psicoativa (F10-F19).

$\mathrm{Na}$ categoria dos antipsicóticos, os medicamentos mais prescritos foram levomepromazina $(34,1 \%)$, risperidona $(32,5 \%)$, clorpromazina $(17,4 \%)$, haloperidol $(14,8 \%)$, olanzapina $(9 \%)$, tioridazina $(8,3 \%)$, flufenazina depot $(6,4 \%)$, quetiapina $(5,1 \%)$, haloperidol depot $(5,2 \%)$ e pimozida (4,5\%) (Figura 2A). Entre os antidepressivos, os mais prescritos foram fluoxetina $(16,1 \%)$, citalopram $(15,4 \%)$, amitriptilina $(9,6 \%)$, clomipramina $(2,5 \%)$, nortriptilina $(0,64 \%)$, imipramina $(0,64 \%)$, venlafaxina $(0,64 \%)$, sertralina $(0,64 \%)$ e mirtazapina $(0,64 \%)$ (Figura $2 \mathrm{~B})$. 

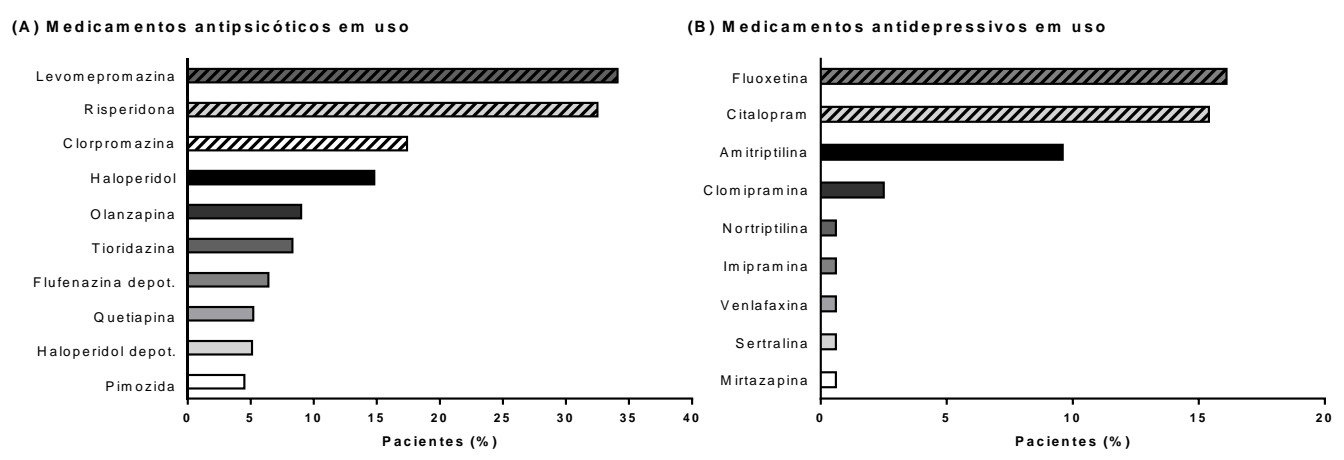

Figura 2 - Relação dos medicamentos antipsicóticos (A) e antidepressivos (B) utilizados, obtida a partir dos prontuários médicos de 155 pacientes psiquiátricos admitidos no CAPS "Esperança" de Recife (PE), no período entre janeiro de 2013 e junho de 2016.

Após a triagem preliminar dos prontuários médicos e traçar o perfil farmacoterapêutico dos usuários do CAPS "Esperança”, 51 pacientes psiquiátricos em regime de tratamento em politerapia foram selecionados e entrevistados. A Tabela 1 resume as principais variáveis demográficas, socioeconômicas e relacionadas ao tratamento e à vida pessoal desta população específica. Os dados revelaram um predomínio do sexo masculino (61\%) em relação ao feminino (39\%), com a faixa etária mais acometida situada entre 35 a 44 anos $(27 \%)$, seguida de 45 a 54 anos (24\%). Também foi observado um predomínio de pacientes solteiros (62\%) e índice de residência urbana de $100 \%$. No que se refere ao grau de escolaridade, a maioria dos pacientes se enquadrou na categoria do ensino fundamental (51\%), seguido pelo ensino médio (37\%). A renda familiar mensal que predominou foi de 1 a 4 salários mínimos (78\%). A maioria dos pacientes entrevistados declarou não exercer qualquer tipo de atividade laboral $(71 \%)$ e atividade física $(74 \%)$, assim como negou o consumo de bebidas alcoólicas (94\%) e tabaco (65\%). 88\% dos pacientes relataram participar de oficinas terapêuticas oferecidas pelo CAPS "Esperança" e 90\% atribuíram conceito bom para a autopercepção de sua saúde. Entre os pacientes entrevistados, $65 \%$ responderam total satisfação quanto às orientações fornecidas pela instituição.

Tabela 1 - Caracterização das variáveis demográficas, socioeconômicas e relacionadas ao tratamento e à vida pessoal de 51 pacientes psiquiátricos selecionados, em regime de tratamento em politerapia, e admitidos no CAPS "Esperança" de Recife (PE), no período entre janeiro de 2013 e junho de 2016.

\begin{tabular}{|lc|}
\hline Variáveis & $\mathbf{n}(\%)$ \\
\hline Gênero & \\
$\quad$ Masculino & $31(61)$ \\
Feminino & $20(39)$ \\
\hline Faixa etária (anos) & \\
18 a 24 & $7(14)$ \\
25 a 34 & $9(18)$ \\
35 a 44 & $14(27)$ \\
45 a 54 & $12(24)$ \\
55 ou mais & $9(18)$ \\
Estado civil & $32(62)$ \\
Solteiro(a) & $12(24)$ \\
Casado(a) & $6(12)$ \\
Divorciado(a) & $1(2)$ \\
Viúvo(a) &
\end{tabular}




\begin{tabular}{|cc}
\hline Residência & continuação \\
Urbana & \\
Rural & $51(100)$ \\
Escolaridade & $0(0)$ \\
Sem escolaridade & \\
Ensino fundamental & $2(4)$ \\
Ensino médio & $26(51)$ \\
Ensino superior & $19(37)$ \\
Renda familiar mensal & $4(8)$ \\
< 1 salário mínimo & \\
1 a 4 salários mínimos & $9(18)$ \\
> salários mínimos & $40(78)$ \\
Atividade laboral & $2(4)$ \\
Sim & \\
Não & $15(29)$ \\
\hline Atividade física & $36(71)$ \\
Sim & $13(26)$ \\
Não & $38(74)$ \\
\hline Uso de álcool & \\
Sim & $3(6)$ \\
Não & $48(94)$ \\
Tabagismo & $18(35)$ \\
Sim & $33(65)$ \\
Não & \\
Participação em oficinas terapêuticas do CAPS & $45(88)$ \\
Sim & $6(12)$ \\
Não & $46(90)$ \\
Autopercepção do estado de saúde & $5(10)$ \\
Boa & $33(65)$ \\
Ruim & $18(35)$ \\
\hline Satisfação quanto às orientações fornecidas pelo CAPS & \\
Boa & \\
Ruim & \\
\hline
\end{tabular}

Em relação às associações medicamentosas, as mais frequentes (acima de 1\%) e de maior importância para a prática clínica estão listadas na Tabela 2. Foram encontrados pelo menos 13 tipos diferentes de associações medicamentosas contendo pelo menos um antipsicótico ou um antidepressivo. O número de medicamentos utilizados por paciente variou de 2 a 6, com uma média de 3,6 medicamentos por paciente. As principais associações encontradas, em ordem decrescente de prevalência, foram aquelas constituídas por: $(i) 1$ antipsicótico típico +1 antipsicótico atípico com prometazina e/ou biperideno $(17,6 \%),($ ii) 2 ou mais antipsicóticos típicos com prometazina e/ou biperideno (11,8\%), (iii) 1 antipsicótico (típico ou atípico) com prometazina e/ou biperideno +1 antidepressivo ISRS $(11,8 \%)$, (iv) 1 antipsicótico típico com prometazina e/ou biperideno $(7,8 \%),(v) 1$ antipsicótico atípico com prometazina e/ou biperideno $(7,8 \%)$, (vi) 2 antipsicóticos típicos +1 antipsicótico atípico com prometazina e/ou biperideno $(7,8 \%)$, (vii) 1 antipsicótico (típico ou atípico) +1 antidepressivo ISRS +1 ATC +1 benzodiazepínico $(7,8 \%$ ), (viii) 1 antidepressivo ISRS +1 ATC +1 benzodiazepínico (5,9\%), (ix) 1 antipsicótico (típico ou atípico) +1 ATC +1 benzodiazepínico $(3,9 \%),(x)$ 1 antipsicótico típico + carbamazepina + lítio ou benzodiazepínico (3,9\%), (xi) 2 antipsicóticos típicos (2,0\%), (xii) 2 antipsicóticos típicos +1 antipsicótico atípico +1 benzodiazepínico $(2,0 \%)$ e (xiii) 1 antipsicótico atípico + lítio +1 benzodiazepínico $(2,0 \%)$. Os demais pacientes receberam outras associações diferentes das mencionadas anteriormente, totalizando $7,9 \%$. 
As reações adversas mais relatadas durante a entrevista pelos pacientes em tratamento através de associações medicamentosas foram os sintomas anticolinérgicos $(64,7 \%)$, ganho de peso $(56,9 \%)$, sintomas extrapiramidais $(50,9 \%)$, sedação $(47,1 \%)$, déficit de memória $(45,1 \%)$, náuseas $(37,2 \%)$, hipotensão ortostática $(35,3 \%)$, fraqueza muscular $(35,3 \%)$ e tontura $(31,4 \%)$.

Tabela 2 - Principais associações medicamentosas utilizadas e reações adversas relatadas por 51 pacientes psiquiátricos selecionados, em regime de tratamento em politerapia, e admitidos no CAPS “Esperança” de Recife (PE), no período entre janeiro de 2013 e junho de 2016.

\begin{tabular}{|c|c|c|c|c|c|c|c|c|c|c|}
\hline \multicolumn{2}{|c|}{$\begin{array}{l}\text { Principais associações } \\
\text { medicamentosas }\end{array}$} & \multicolumn{9}{|c|}{$\begin{array}{c}\text { Principais reações adversas relatadas } \\
\text { Freq }(\%)\end{array}$} \\
\hline Frec & $(\%)$ & EAN & GPE & SEP & SED & DME & NAU & HOR & FMU & TON \\
\hline $\begin{array}{c}\text { 1. } 1 \text { Antipsicótico T } \\
+ \text { BIP e/ou } \\
\text { PRO }\end{array}$ & $4(7,8)$ & $3(5,9)$ & $0(0,0)$ & $3(5,9)$ & $2(3,9)$ & $2(3,9)$ & $1(2,0)$ & $1(2,0)$ & $2(3,9)$ & $2(3,9)$ \\
\hline $\begin{array}{c}\text { 2. } 1 \text { Antipsicótico A } \\
+ \text { BIP e/ou } \\
\text { PRO }\end{array}$ & $4(7,8)$ & $3(5,9)$ & $2(3,9)$ & $2(3,9)$ & $3(5,9)$ & $3(5,9)$ & $1(2,0)$ & $0(0,0)$ & $2(3,9)$ & $2(3,9)$ \\
\hline 3. 2 Antipsicótico T & $1(2,0)$ & $1(2,0)$ & $1(2,0)$ & $0(0,0)$ & $0(0,0)$ & $1(2,0)$ & $0(0,0)$ & $1(2,0)$ & $0(0,0)$ & $1(2,0)$ \\
\hline $\begin{array}{c}\text { 4. } \geq 2 \text { Antipsicóticos T } \\
+ \text { BIP e/ou } \\
\text { PRO }\end{array}$ & $6(11,8)$ & $4(7,8)$ & $5(9,8)$ & $4(7,8)$ & $2(3,9)$ & $3(5,9)$ & $3(5,9)$ & $1(2,0)$ & $2(3,9)$ & $2(3,9)$ \\
\hline $\begin{array}{l}\text { 5. } 1 \text { Antipsicótico T } \\
+1 \text { Antipsicótico A } \\
+ \text { BIP e/ou PRO }\end{array}$ & $9(17,6)$ & $5(9,8)$ & $5(9,8)$ & $5(9,8)$ & $6(11,8)$ & $1(2,0)$ & $5(9,8)$ & $3(5,9)$ & $4(7,8)$ & $0(0,0)$ \\
\hline $\begin{array}{l}\text { 6. } 2 \text { Antipsicóticos T } \\
+1 \text { Antipsicótico A } \\
+ \text { BIP e/ou PRO }\end{array}$ & $4(7,8)$ & $2(3,9)$ & $2(3,9)$ & $3(5,9)$ & $1(2,0)$ & $1(2,0)$ & $2(3,9)$ & $1(2,0)$ & $0(0,0)$ & $1(2,0)$ \\
\hline $\begin{array}{c}\text { 7. } 2 \text { Antipsicótico T } \\
+1 \text { Antipsicótico A + } \\
\text { BDZ }\end{array}$ & $1(2,0)$ & $0(0,0)$ & $1(2,0)$ & $1(2,0)$ & $0(0,0)$ & $0(0,0)$ & $0(0,0)$ & $0(0,0)$ & $0(0,0)$ & $0(0,0)$ \\
\hline $\begin{array}{l}\text { 8. } 1 \text { Antipsicótico (T } \\
\text { ou A) + BIP e/ou } \\
\text { PRO + } 1 \text { ISRS }\end{array}$ & $6(11,8)$ & $3(5,9)$ & $4(7,8)$ & $3(5,9)$ & $3(5,9)$ & $2(3,9)$ & $2(3,9)$ & $4(7,8)$ & $2(3,9)$ & $2(3,9)$ \\
\hline $\begin{array}{c}\text { 9. 1 Antipsicótico (T } \\
\text { ou A) }+1 \text { ATC + } \\
\text { BDZ }\end{array}$ & $2(3,9)$ & $2(3,9)$ & $1(2,0)$ & $0(0,0)$ & $0(0,0)$ & $1(2,0)$ & $0(0,0)$ & $0(0,0)$ & $1(2,0)$ & $1(2,0)$ \\
\hline $\begin{array}{c}1 \text { Antipsicótico (T } \\
\text { ou A) + 1 ISRS + } \\
1 \text { ATC + BDZ }\end{array}$ & $4(7,8)$ & $4(7,8)$ & $3(5,9)$ & $3(5,9)$ & $1(2,0)$ & $3(5,9)$ & $2(3,9)$ & $3(5,9)$ & $1(2,0)$ & $2(3,9)$ \\
\hline $\begin{array}{l}\text { 11. Antipsicótico T } \\
+\mathrm{CBZ}+\mathrm{Li} \\
\text { ou BDZ }\end{array}$ & $2(3,9)$ & $1(2,0)$ & $0(0,0)$ & $2(3,9)$ & $1(2,0)$ & $1(2,0)$ & $0(0,0)$ & $0(0,0)$ & $2(3,9)$ & $1(2,0)$ \\
\hline $\begin{array}{c}\text { 12. } 1 \text { Antipsicótico A } \\
+\mathrm{Li} \\
+\mathrm{BDZ}\end{array}$ & $1(2,0)$ & $0(0,0)$ & $0(0,0)$ & $0(0,0)$ & $0(0,0)$ & $1(2,0)$ & $0(0,0)$ & $0(0,0)$ & $0(0,0)$ & $0(0,0)$ \\
\hline $\begin{array}{l}\text { 13. ISRS + } \\
1 \mathrm{ATC}+ \\
\mathrm{BDZ}\end{array}$ & $3(5,9)$ & $3(5,9)$ & $2(3,9)$ & $0(0,0)$ & $1(2,0)$ & $2(3,9)$ & $2(3,9)$ & $3(5,9)$ & $1(2,0)$ & $1(2,0)$ \\
\hline Outras & $4(7,9)$ & $2(3,9)$ & $3(5,9)$ & $0(0,0)$ & $4(7,9)$ & $2(3,9)$ & $1(2,0)$ & $1(2,0)$ & $1(2,0)$ & $1(2,0)$ \\
\hline Total & $51(100)$ & $33(64,7)$ & $29(56,9)$ & $26(50,9)$ & $24(47,1)$ & $23(45,1)$ & $19(37,2)$ & $18(35,3)$ & $18(35,3)$ & $16(31,4)$ \\
\hline
\end{tabular}

Abreviaturas: $\quad$ Freq=frequência, $\quad \mathrm{T}=$ =típico, $\mathrm{A}=$ atípico, $\mathrm{BIP}=$ biperideno, $\mathrm{PRO}=$ prometazina, BDZ=benzodiazepínico, ISRS=inibidor seletivo da recaptação de serotonina, ATC=antidepressivo tricíclico, $\mathrm{CBZ}=$ carbamazepina, Li=Lítio, EAN=efeitos anticolinérgicos, GPE=ganho de peso, $\mathrm{SEP}=$ sintomas extrapiramidais, $\mathrm{SED}=$ sedação, $\mathrm{DME}=$ déficit de memória, $\mathrm{HOR}=$ hipotensão ortostática, $\mathrm{FMU}=$ fraqueza muscular, NAU=náuseas, TON=tontura. 


\section{Discussão}

A análise dos prontuários médicos de pacientes psiquiátricos admitidos entre $2013 \mathrm{e}$ 2016 no CAPS "Esperança” de Recife constatou um predomínio importante de usuários do sexo feminino sobre o masculino. Estes resultados endossam aqueles relatados por Paula (15), Gomes e Bellettine (16) e Martins e colaboradores (17), que mostraram que a maioria dos usuários de serviços substitutivos é representada pela população feminina. A maioria dos usuários admitidos no CAPS "Esperança" encontra-se na faixa etária situada entre 35 e 44 anos, seguido por pacientes com idade entre 45 e 54 anos. Estes resultados corroboram a análise epidemiológica realizada por Miranda, Tarasconi e Scortegagna (2008), que apontaram uma maior procura pelo atendimento em saúde mental na faixa etária situada entre 30 e 49 anos, correspondendo a 41,8\% (18). Percebe-se, assim, que os transtornos psiquiátricos vêm atingindo a população economicamente ativa ou produtiva (2). Observou-se nos prontuários analisados que a maioria dos diagnósticos psiquiátricos foi referente a quadros de esquizofrenia e transtornos psicóticos relacionados. Estas doenças estão amplamente associadas à considerável inaptidão, comprometendo a realização educativa e ocupacional, além de estarem associadas a maior probabilidade (2 a 2,5 vezes) de óbito precoce do que a população geral (2).

No presente estudo, os dados dos prontuários mostraram também que a categoria de medicamentos mais utilizada pelos pacientes do CAPS "Esperança" foram os antipsicóticos, estando em consonância ao elevado número de pacientes com diagnóstico de esquizofrenia e transtornos psicóticos relacionados. Estes transtornos mentais são primariamente tratados com medicamentos antipsicóticos, embora estes fármacos, especialmente os de $2^{\text {a }}$ geração, também possam ser úteis no tratamento de outras condições psiquiátricas distintas, como por exemplo, nos transtornos do humor e de ansiedade $(9,29)$. A segunda e a terceira classe de medicamentos mais utilizados no CAPS "Esperança" foram os antidepressivos e ansiolíticos benzodiazepínicos, respectivamente, reflexo do número elevado de pacientes com diagnósticos de transtornos de humor em comorbidade a quadros de ansiedade. Entre os medicamentos antipsicóticos, os mais prescritos foram levomepromazina e risperidona. A levomepromazina é um antipsicótico de $1^{a}$ geração (típico) utilizado como coadjuvante em quadros de agitação e excitação psicomotora, face ao seu perfil de ação sedativa. $\mathrm{O}$ segundo fármaco mais utilizado foi a risperidona, um antipsicótico de $2^{a}$ geração (atípico). Este fármaco está associado a menor possibilidade de reações adversas relacionadas ao sistema extrapiramidal quando comparado à levomepromazina, além de apresentar maior tolerabilidade pelos pacientes em relação aos distúrbios motores, embora esteja associada ao considerável ganho de peso. Consequentemente, ao ganho ponderal está associado um maior risco de doenças associadas à obesidade, tais como a diabetes mellitus, hipertensão, doenças coronarianas e síndrome metabólica $(9,19)$.

Entre os antidepressivos, o fármaco mais utilizado pelos pacientes foi fluoxetina, um ISRS, seguido do citalopram (também ISRS) e amitriptilina (um ATC). A categoria dos ISRS não apresenta diferença em relação à eficácia quando comparada aos ATC, entretanto, estes últimos são responsáveis pela maior variedade de reações adversas, estando associados a diferentes taxas de desistência ao tratamento quando comparados aos ISRS (20). Consequentemente, a presente pesquisa mostrou uma preferência pelo uso dos ISRS em substituição aos ATC e IMAO, face ao melhor perfil de segurança, menor variedade de reações adversas e maior taxa de tolerabilidade dos primeiros em relação aos últimos. Contudo, os ATC continuam sendo fármacos bastante utilizados na prática clínica devido ao custo reduzido, eficácia comprovada e como opção de tratamento para pacientes refratários aos medicamentos de primeira linha (21). A importância dos ATC 
na prática clínica também foi observada na presente pesquisa com o uso considerável da amitriptilina, a terceira opção de fármaco mais utilizada entre os antidepressivos.

$\mathrm{Na}$ segunda etapa deste estudo, foram analisados 51 pacientes em regime de tratamento em politerapia. Os dados demográficos e socioeconômicos que caracterizam o perfil destes pacientes mostraram um predomínio do sexo masculino (61\%) e maior concentração de pacientes na faixa etária economicamente ativa (35 a 54 anos), com índice de residência urbana de $100 \%$. Em relação ao estado civil, $63 \%$ dos entrevistados relataram estar solteiros, seguido do segundo maior percentual representado pelos casados (24\%). Com relação à escolaridade, metade dos pacientes analisados não concluiu o primeiro grau que, segundo Lima e colaboradores (1999), torna o portador de transtorno psiquiátrico mais vulnerável a situações de desemprego e baixo rendimento mensal (22). Consequentemente, o baixo nível de escolaridade da população acarreta dificuldades na leitura e interpretação das informações sobre os medicamentos, situação que é agravada em pacientes submetidos ao tratamento em politerapia, aumentando o risco do uso incorreto e de ocorrência de reações adversas aos medicamentos. Observouse um predomínio de pacientes com baixa renda, com rendimento familiar mensal de um a quatro salários mínimos (78\%), seguido daqueles que recebem até um salário mínimo $(18 \%)$. Os dados também mostraram que a maioria dos pacientes entrevistados estão afastados de qualquer tipo de atividade física e laboral (71 e 75\%, respectivamente), situação que pode estar relacionada, em parte, às reações adversas associadas ao grande número de fármacos utilizados, especialmente a sedação e o ganho ponderal discutidos na sequência. A maioria dos pacientes relatou participar de oficinas terapêuticas oferecidas pelo CAPS "Esperança" e atribuíram conceito "bom" para a autopercepção de sua saúde (88 e $90 \%$, respectivamente), com índice de satisfação total de $65 \%$ quanto às orientações fornecidas pela instituição.

Entre as associações medicamentosas analisadas, pode-se notar que a duplicidade no uso de fármacos do mesmo grupo terapêutico não ocorreu de forma expressiva com a categoria dos antidepressivos, em distinção a associação comum entre dois antipsicóticos. As associações medicamentosas que predominaram foram aquelas constituídas por dois antipsicóticos (típico + atípico) com biperideno e/ou prometazina, seguida da associação entre 2 ou mais antipsicóticos típicos com biperideno e/ou prometazina, 1 antipsicótico (típico ou atípico) + biperideno e/ou prometazina com 1 antidepressivo ISRS e 1 antipsicótico típico + biperideno e/ou prometazina. É importante mencionar que a literatura médica preconiza a realização do tratamento da esquizofrenia ou quadros psicóticos relacionados através da monoterapia ao invés do uso de associações medicamentosas, já que não há evidências clínicas de que a combinação de antipsicóticos traga algum tipo de benefício adicional em termos de redução dos sintomas da esquizofrenia (23). Assim, o tratamento ideal deve ser realizado com um medicamento antipsicótico por vez, de acordo com o perfil de segurança e tolerabilidade do paciente.

Em relação às principais reações adversas relatadas pelos pacientes, os sintomas anticolinérgicos periféricos (como por exemplo, boca seca, visão turva, constipação e/ou retenção urinária) ganharam destaque, correspondendo a 64,7\% das associações medicamentosas encontradas. Embora descritas entre a maioria das associações, a maior prevalência de sintomas anticolinérgicos ocorreu em pacientes fazendo uso de combinações constituídas por 1 antipsicótico típico +1 antipsicótico atípico com biperideno e/ou prometazina ( 5 em 9 pacientes, 9,8\%), 2 ou mais antipsicóticos típicos com biperideno e/ou prometazina (4 em 6 pacientes, 7,8\%), ou 1 antipsicótico (típico ou atípico) +1 ISRS +1 ATC +1 benzodiazepínico (todos os 4 pacientes, 7,8\%). Em situações de tratamentos realizados em monoterapia, a maioria dos sintomas anticolinérgicos são previsíveis e considerados de natureza leve. Entretanto, no âmbito 
do CAPS onde a politerapia é muito comum, a utilização de fármacos com ações anticolinérgicas adicionais, como por exemplo, dois ou mais antipsicóticos providos de ações anticolinérgicas, ou ainda, um ou mais antipsicóticos com ação anticolinérgica combinados à prometazina e/ou biperideno, aumenta o risco de morbimortalidade, especialmente a incidência de efeitos sobre o trato gastrointestinal, como constipação severa, íleo paralítico, isquemia mesentérica e óbito devido à perfuração intestinal e sepse, além da própria intoxicação anticolinérgica que pode ser fatal (24-25). Além disso, a associação de um ISRS com um ATC também identificada na presente pesquisa, muito embora possa ter sido utilizada visando a potencialização do tratamento psiquiátrico, aumenta o risco de ocorrência de reações adversas. A fluoxetina (um ISRS), por exemplo, eleva as concentrações plasmáticas dos ATC, intensificando os sintomas anticolinérgicos. Além disso, a combinação de fluoxetina com um ATC aumenta a possibilidade de desenvolvimento de cardiotoxicidade e síndrome serotoninérgica, ambas das quais podendo ser fatais (26). Adicionalmente, a gravidade destes sintomas aumenta ainda mais com o uso concomitante de antipsicóticos (27), associação também identificada na presente pesquisa.

A segunda reação adversa mais relatada pelos pacientes foi o ganho ponderal, constituindo 56,9\% dos casos. Embora o ganho ponderal ocasionado por determinados fármacos possa ser desejável em casos específicos de pacientes com transtornos psiquiátricos, ele pode acarretar secundariamente diversos problemas de saúde, entre eles, a obesidade, diabetes, hipertensão, dislipidemias e, consequentemente, evoluir para a síndrome metabólica. A síndrome metabólica é uma doença altamente preocupante e torna-se um risco imediato ao paciente com transtorno mental devido à pouca prática de atividade física e a inexistência de um protocolo ideal para o devido manejo desta condição clínica na psiquiatria. Esta condição clínica pode ser resultante tanto do uso de antidepressivos quanto de antipsicóticos, em parte, devido ao bloqueio dos receptores histaminérgicos $\mathrm{H}_{1}$ e serotoninérgicos $5-\mathrm{HT}_{2 \mathrm{C}}$ a nível hipotalâmico $(8,19,23)$. Embora não seja necessária a retirada do(s) medicamento(s) para estes casos, faz-se necessária a devida orientação e monitorização do paciente (28). Adicionalmente, o bloqueio dos receptores histaminérgicos $\mathrm{H}_{1}$ é a causa habitual da sedação (29), sendo a clorpromazina o antipsicótico típico mais sedativo entre os utilizados. A sedação foi identificada como a quarta reação adversa mais comum entre os pacientes do CAPS "Esperança", sendo responsável por causar diversos problemas ao paciente, em decorrência do prejuízo em atividades rotineiras como estudar, trabalhar, participar de compromissos sociais e realizar exercícios físicos, aspectos que também contribuem para o ganho de peso e desenvolvimento da síndrome metabólica. Consequentemente, tanto a sedação quanto o ganho de peso ocasionados por determinadas associações medicamentosas são fatores que influenciam negativamente a qualidade de vida do usuário do CAPS, razões que justificam o elevado índice de pacientes psiquiátricos que declararam, na presente pesquisa, não exercer qualquer tipo de atividade física (74\% e $71 \%$, respectivamente).

É digno de nota mencionar que algumas associações medicamentosas são consideradas importantes e utilizadas justamente para minimizar eventuais reações adversas ocasionadas por um determinado fármaco, ou até mesmo para beneficiar o estado clínico do paciente. A associação com biperideno, por exemplo, devido à sua ação anticolinérgica, permite controlar os sintomas extrapiramidais ocasionadas por antipsicóticos (30), descritos na presente pesquisa como a terceira reação adversa mais comum entre as associações medicamentosas. Já a associação de antipsicóticos com prometazina é amplamente utilizada na prática clínica para a promoção da sedação, consequentemente, a sedação dentro deste contexto não é considerada uma reação adversa. A ação anti-histaminérgica e anticolinérgica promovida tanto pela 
levomepromazina quanto pela prometazina reduz a agitação psicomotora, controla a agressividade e insônia quando presentes, além de minimizar os sintomas extrapiramidais ocasionados pelo próprio antipsicótico $(19,29)$.

$\mathrm{Na}$ presente pesquisa, tanto a prometazina quanto o biperideno estiveram presentes em 6 das 13 associações medicamentosas utilizadas pelos pacientes, constituindo quase a metade de todas as associações encontradas. Entretanto, foi observado que uma grande parte destes pacientes, inclusive aqueles utilizando prometazina e biperideno concomitantemente, ainda continuaram apresentando algum tipo de sintoma extrapiramidal, possivelmente em decorrência da dose elevada do antipsicótico utilizado ou da própria combinação de dois ou mais antipsicóticos, razões que aumentam a possibilidade de ocorrência das alterações motoras. Os sintomas extrapiramidais mais comuns incluem o parkinsonismo farmacológico, distonias, acatisia e discinesia tardia. Com exceção da discinesia tardia, os demais geralmente ocorrem no início do tratamento, embora possam persistir durante todo o período de manutenção (19). Entre as hipóteses mais aceitas que justifiquem o surgimento dos sintomas extrapiramidais se destacam: i) a taxa de ocupação dos receptores D2/5-HT2 pelo antipsicótico, com a maior ocupação D2 e/ou menor ocupação 5-HT2 aumentando a incidência dos sintomas motores, ii) a velocidade de dissociação dos receptores $\mathrm{D} 2$, com os antipsicóticos que rapidamente se dissociam do receptor apresentando menor potencial em promover alterações motoras, iii) a hiperatividade colinérgica secundária ao bloqueio D2 ocasionado pelo antipsicótico, e iv) no caso particular da discinesia tardia devido ao bloqueio contínuo ocasionado pelo uso crônico do antipsicótico, vem sendo proposto uma hipersensibilidade dos receptores D2, deficiência GABAérgica, mudanças plásticas em redes dopaminérgicas estriatais e/ou neurotoxicidade (19,31-32). Em casos de intolerância aos sintomas extrapiramidais, mesmo após o ajuste da dose do antipsicótico ou após a combinação com prometazina ou biperideno, está indicada a substituição por outro antipsicótico com menor perfil de sintomas extrapiramidais, e não a associação de antipsicóticos que, como mencionado anteriormente, não traz nenhum benefício adicional ao paciente.

Outra reação adversa relatada pelos pacientes foi a hipotensão ortostática, caracterizada pela queda abrupta da pressão arterial resultante da mudança rápida da postura supina para a posição vertical. Ela ocorre devido ao bloqueio dos receptores alfa-1 adrenérgicos pelos antipsicóticos e antidepressivos (33). A queda na pressão arterial pode ser assintomática ou levar o indivíduo a apresentar tontura, dor de cabeça, tremores, turvamento da visão, tremores, astenia, palpitação, síncope, confusão mental e quedas (34). Embora os pacientes desenvolvam tolerância à hipotensão postural, os mesmos devem ser advertidos quanto à sua ocorrência, especialmente no início do tratamento, e devidamente orientados a levantar-se mais lentamente quando estiverem deitados ou sentados, não ingerir álcool e cafeína e desenvolver o hábito de ingerir bastante água durante o dia.

Aproximadamente um terço dos pacientes com transtornos depressivos não respondem ao tratamento com os antidepressivos convencionais, e aqueles respondedores geralmente levam semanas a meses para obter um efeito terapêutico significativo (35). Diversos fatores contribuem para a refratariedade farmacológica do paciente psiquiátrico, um deles é a baixa aderência ao tratamento farmacológico devido à presença de reações adversas. Existem algumas estratégias utilizadas em casos de refratariedade, como por exemplo, aumento da dose do fármaco, combinação de antidepressivos ou a substituição do antidepressivo por outra categoria. No caso de pacientes esquizofrênicos refratários, a clozapina é a única opção disponível atualmente, sendo considerado o tratamento padrãoouro para estes casos. Embora a clozapina reduza a sintomatologia aguda e o risco de suicídio dos pacientes, este fármaco também está associado ao ganho de peso e síndrome 
metabólica, e tem uma grande limitação quanto à possibilidade de desenvolvimento de agranulocitose, que pode ser fatal (29,36-37). Entretanto, não foi identificado no CAPS "Esperança" qualquer paciente fazendo uso de clozapina durante o período analisado.

Apesar da baixa prevalência de alcoolismo descrita entre os pacientes analisados, uma associação bastante considerável (35\%) foi encontrada entre o tabagismo e pacientes com esquizofrenia. Esta última associação, já bem descrita na literatura científica, contribui negativamente para a saúde física e mental e leva a prejuízos na vida social e econômica destes pacientes (38). Sustentada pela hipótese dos efeitos da nicotina sobre o aumento na liberação de dopamina em neurônios dopaminérgicos, a nicotina alivia os sintomas extrapiramidais causados pelas drogas antipsicóticas. Além disso, a nicotina aumenta os níveis de dopamina em neurônios do córtex pré-frontal, o que melhora os sintomas negativos da esquizofrenia (38). Entretanto, apesar dos aparentes benefícios, esta prática prejudica a apresentação e gravidade do transtorno psiquiátrico e interfere negativamente na eficácia do tratamento farmacológico.

A ocorrência de reações adversas a medicamentos no âmbito dos CAPS, portanto, deteriora a saúde do paciente psiquiátrico e causa custos adicionais ao sistema de saúde. Embora difícil de evitá-las na íntegra, o seu reconhecimento e o adequado manejo minimizam os riscos das reações adversas e os custos assistenciais.

\section{Limitações do estudo}

As potenciais reações adversas analisadas se limitam apenas aos medicamentos prescritos nos prontuários cuja utilização foi confirmada pelo paciente durante a entrevista e pela equipe multidisciplinar, desconsiderando, portanto, o possível uso de outros medicamentos não psiquiátricos associados. Neste estudo não é possível afirmar que tais reações adversas tenham relação direta com os medicamentos utilizados e se algumas destas reações (por exemplo, sedação ou ganho de peso) foram almejadas pelo corpo clínico como parte complementar do tratamento de um determinado paciente. Também não foi possível estudar o critério de escolha dos medicamentos prescritos pela equipe médica, bem como a evolução clínica dos pacientes ao longo do período analisado.

\section{Conclusões}

Este estudo revelou o potencial elevado de reações adversas relacionadas ao uso de antipsicóticos ou antidepressivos e fármacos associados no âmbito do CAPS "Esperança" de Recife, possivelmente relacionadas ao regime de tratamento em politerapia. Algumas das reações adversas são de grande relevância clínica e podem comprometer o bem-estar e a adesão do paciente ao tratamento. Desta forma, a participação ativa do profissional farmacêutico no acompanhamento terapêutico é fundamental para garantir o uso racional dos medicamentos e melhorar a qualidade de vida do paciente psiquiátrico, permitindo a ele maior reinserção social.

\section{Conflito de Interesses}

Os autores declaram que não existe conflito de interesse e que todos os gastos ocorridos com esta pesquisa foram realizados com auxílio da Universidade Federal do Pernambuco e dos próprios pesquisadores envolvidos.

\section{Referências}


1. Santos ÉG dos, Siqueira MM de. Prevalência dos transtornos mentais na população adulta brasileira: uma revisão sistemática de 1997 a 2009. J Bras Psiquiatr 2010; 59(3): 238-246.

2. WHO. Mental disorders. 2018 [cited 2019 Feb 12]. Available from: https://www.who.int/en/newsroom/fact-sheets/detail/mental-disorders.

3. American Psychiatric Association. Manual Diagnóstico e Estatístico de Transtornos Mentais. DSM-5. 5th ed. Porto Alegre: Artmed; 2014.

4. BRASIL. Ministério da Saúde. Portaria $n^{\circ}$ 336, de 19 de fevereiro de 2002. Define e estabelece diretrizes para o funcionamento dos Centros de Atenção Psicossocial. Brasília, DF: Ministério da Saúde; 2002. Disponível em: < http:// www.saude.gov.br >. Acesso em: 03 nov. 2017.

5. Felipe RL, Martins DA, Cardoso RA, Franqueiro EPM, Garivl MP, Lucchese R. Análise descritiva dos fármacos utilizados por pacientes atendidos no Centro de Atenção Psicossocial (CAPS), Catalão - GO. In: v. 5, ed. Reunião Anual de Ciências. Uberlândia: E-RAC; 2015:1-11.

6. Freire EC, Feijó CFC, Fonteles MMF, Soares JES, Carvalho TMJP. Adesão ao tratamento medicamentoso em usuários com transtorno do humor de centro de atenção psicossocial do nordeste do Brasil. Rev Cien Farm Basica Apl 2013; 3:565-570.

7. Souza FG de M. Tratamento da depressão. Rev Bras Psiquiatr 1999; 21(1): 18-23.

8. De Hert M, Schreurs V, Vancampfort D, Van Winkel R. Metabolic syndrome in people with schizophrenia: a review. World Psychiatry 2009; 8(1): 15-22.

9. Alves CRR, Silva MTA. A esquizofrenia e seu tratamento farmacológico. Estud Psicol 2001; 18(1): $12-22$.

10. Ivama AM, Noblat L, Castro MS, Jaramillo NM, de Oliveira NVBV, Rech N. Atenção Farmacêutica No Brasil: Trilhando Caminhos. Relatório 2001-2002. Brasília: Organização Pan-Americana da Saúde; 2002.

11. Galán A, Fernández P. Autovaloración social, económica y profesional del farmacéutico comunitario. Rev la OFIL 1991; 1(5): 298-202.

12. Santos JHP, Silva TP. A importância da atenção farmacêutica nos Centros de Atenção Psicossocial (CAPS) [monography]. Universidade Estadual de Goiás; 2011.

13. Alves DA dos S. A importância do farmacêutico no Centro de Atenção Psicossocial (CAPS) [cited 2019 Feb 11]. Available from: https://www.webartigos.com/artigos/a-importancia-do-farmaceuticono-centro-de-atencao-psicossocial-caps/118999.

14. BRASIL. Ministério da Saúde. Resolução n. 466, de 12 de Dezembro de 2012. Brasília; 2012.

15. Paula CTC. Perfil epidemiológico dos usuários de um Centro de Atenção Psicossocial na cidade de Recife. Cad Bras Saúde Ment 2010; 2(4-5): 94-105.

16. Gomes KM, Bellettine F. Perfil dos usuários do centro de atenção psicossocial e do programa de saúde mental no município de Orleans - SC. Cad Bras Saúde Ment J Ment Heal 2013; 5(12): 161-175.

17. Martins MAC, Hostalácio AM, Silva VLQ, Rosa W de AG, Almeida DA de. Perfil dos pacientes atendidos em um centro de atenção psicossocial, em Passos - MG. Rev Iniciação Científica da Lib 2014; 4(2): 121-134.

18. Miranda CA, Tarasconi CV, Scortegagna SA. Estudo epidêmico dos transtornos mentais. Avaliação Psicológica 2008; 7(2): 249-257.

19. Brunton LL, Lazo JS, Parker KL. Goodman \& Gilman: As Bases Farmacológicas Da Terapêutica. 10th ed. Rio de Janeiro: McGraw-Hill; 2005.

20. Wannmacher L. Abordagem da depressão maior em idosos: medidas não medicamentosas e medicamentosas. Uso Racion Medicam Fundam em Condutas Ter e nos Macroprocessos da Assist Farm 2016; 1(1): 1-10.

21. Gervasoni N, Aubry JM, Gex-Fabry M, Bertschy G, Boldolfi G. Is there a place for tricyclic antidepressants and subsequent augmentation strategies in obtaining remission for patients with treatment resistant depression? Pharmacol Res 2009; 59(3): 202-6.

22. Lima, MS, Soares, BGO, Mari, JJ. Saúde e doença mental em Pelotas, RS: dados de um estudo populacional. Rev Psiquiatr Clínica 1999; 26(5): 225-235.

23. Louzã Neto MR, Elkis H. Psiquiatria Básica. 2nd ed. Porto Alegre: Artmed; 2007.

24. Peyrière H, Roux C, Ferard C, Deleau N, Kreft-Jais C, Hillaire-Buys D, Boulenger JP, Blayac JP. 
Antipsychotics-induced ischaemic colitis and gastrointestinal necrosis: a review of the French pharmacovigilance database. Pharmacoepidemiol Drug Saf 2009; 18(10): 948-55.

25. Nielsen J, Meyer JM. Risk Factors for Ileus in Patients with Schizophrenia. Schizophr Bull 2012; 38(3): 592-598.

26. Westermeyer J. Fluoxetine-induced tricyclic toxicity: extent and duration. J Clin Pharmacol 1991; 31(4): 388-92.

27. Khasawneh FT, Shankar GS. Minimizing cardiovascular adverse effects of atypical antipsychotic drugs in patients with schizophrenia. Cardiol Res Pract 2014; 273060.

28. Teixeira PJR, Rocha FL. Associação entre síndrome metabólica e transtornos mentais. Rev Psiquiatr Clínica 2007; 34(1): 28-38.

29. Sadock BJ, Sadock VA. Manual de farmacologia psiquiátrica de Kaplan e Sadock. Rev Bras Psiquiatr 2002; 24(2): 103-104.

30. Baroza PS, Silva DA da. Medicamentos Antidepressivos e Antipsicóticos Prescritos no Centro de Atenção Psicossocial (CAPS) do Município de Porciúncula - RJ. Acta Biomed Bras 2012; 3(1): 85-97.

31. Caroff SN, Hurford I, Lybrand J, Campbell EC. Movement Disorders Induced by Antipsychotic Drugs: Implications of the CATIE Schizophrenia Trial. Neuro Clin 2011; 29(1):127-viii.

32. Ward KM, Citrome L. Antipsychotic-Related Movement Disorders: Drug-Induced Parkinsonism vs. Tardive Dyskinesia - Key Differences in Pathophysiology and Clinical Management. Neurol Ther 2018; 7(2): 233-248.

33. Moreno RA, Moreno DH, Soares MB de M. Psicofarmacologia de antidepressivos. Rev Bras Psiquiatr 1999; 21(suppl 1): 24-40.

34. Lopes LS, Mürrer G, Lima NCP de, Grizante P, Valente M. Hipotensão ortostática em pacientes idosos ambulatoriais. Arq Médicos do ABC 2007; 32(1): 17-20.

35. De Carlo V, Calati R, Serretti A. Socio-demographic and clinical predictors of non-response/nonremission in treatment resistant depressed patients: A systematic review. Psychiatry Res 2016; 240 : 421-430.

36. Lundblad W, Azzam PN, Gopalan P, Ross CA, PharmD. Medical management of patients on clozapine: A guide for internists. J Hosp Med 2015; 10(8): 537-43.

37. Nebhinani N, Grover S, Chakrabarti S, Kate N, Avasthi A. A longitudinal study of change in prevalence of metabolic syndrome and metabolic disturbances 3 months after Clozapine therapy. J Mental Health Hum Behav 2013; 18(1): 9-17.

38. Moreira EC, Soares HG. Dependência de nicotina em pacientes com esquizofrenia: etiologia e tratamento. J Bras Psiquiatr 2003; 52(4): 251-257. 